Chronic Obstructive Pulmonary Diseases: Journal of the COPD Foundation

\title{
Review
}

\section{A Decade of National Heart, Lung, and Blood Institute Programs Supporting COPD Research and Education}

Lisa Postow, PhD, ${ }^{1}$ Antonello Punturieri, MD, PhD, ${ }^{1}$ Thomas L. Croxton, PhD, MD, ${ }^{1}$ Gail G. Weinmann, MD, ${ }^{1}$ and James P. Kiley, $\mathrm{PhD}^{1}$

\section{Abstract}

The past decade of research in chronic obstructive pulmonary disease (COPD) has seen a new age of understanding both pathogenic mechanisms and clinical manifestations of the disease. The National Heart, Lung, and Blood Institute (NHLBI) has helped guide this progress with a series of initiatives to stimulate COPD research in various ways. These initiatives were designed to promote a precision medicine approach to treating COPD, one that takes advantage of targeting particular molecular pathways and the individual pathobiologies of the diversity of COPD patients. This review describes the strategic objectives of these initiatives, as well as some of their observed and anticipated outcomes. In addition, we address parallel steps NHLBI has taken to promote COPD awareness among the public. As we look toward the immediate future of COPD research and education, we see a time of great progress in terms of understanding and treatment. Furthermore, while this remains a debilitating and disturbingly prevalent disease, as NHLBI looks even farther ahead, we envision emerging efforts toward COPD prevention.

\begin{abstract}
Abbreviations: National Institutes of Health, NIH; National Heart, Lung and Blood Institute, NHLBI; alpha-1 antitrypsin deficiency, A1AT; research project grant, RO1; Lung Tissue Research Consortium, LTRC; computed tomographic, CT; interstitial lung disease, ILD; lung volume reduction surgery, LVRS; funding opportunity announcement, FOA; Specialized Center of Clinically-Oriented Research, SCCOR; Centers for Advanced Diagnostic and Experimental Therapeutics in Lung Disease, CADET; Lung Genomics Research Consortium, LGRC; American Recovery and Reinvestment Act, ARRC; SubPopulations and Intermediate Outcome Measures in COPD Study, SPIROMICS; Genomic Research in Alpha-1 Antitrypsin Deficiency and Sarcoidosis, GRADS; COPD Genetic Epidemiology, COPDGene; Mutlti-ethnic Study of Atherosclerosis, MESA; request for applications, RFA; National Emphysema Treatment Trial, NETT; Lung Health Study, LHS; Feasibililty of Retinoids for the Treatment of Emphysema, FORTE; COPD Clinical Research Network, CCRN; Long-Term Oxygen Treatment Trial, LOTT; Learn More Breathe Better campaign, LMBB; Behavioral Risk Factors Surveillance System, BRFSS

Funding Support: Not applicable

Date of Acceptance: March 10, 2014

Citation: Postow L, Punturieri A, Croxton TL, Weinmann GG, Kiley JP. A decade of National Heart, Lung, and Blood Institute Programs supporting COPD research and education. J COPD F. 2014; 1(1):64-72.doi: http://dx.doi.org/10.15326/jcopdf.1.1.2014.0123
\end{abstract}

1 Division of Lung Diseases

National Heart, Lung, and Blood Institute

National Institutes of Health

Bethesda, MD 20892-7952

\section{Address correspondence to:}

James P. Kiley, Ph.D

Ph:301-435-0203

kileyj@nhlbi.nih.gov

\section{Keywords:}

National Heart, Lung, and Blood Institute; National Institutes of Health; COPD; request for applications; funding opportunity announcement; initiative; subpopulations; genomics; grant resource; biomarkers; education

\section{Introduction}

Geologists divide the past into periods - intervals of relative stability in the earth's characteristics that are often punctuated by brief, even cataclysmic, transitions. For example, the Cretaceous period with its large dinosaurs was ended by an asteroid, leading to the rise of mammals during the subsequent Paleogene period. The history of chronic obstructive pulmonary disease (COPD) has followed a similar pattern, with periods of slow evolution interrupted by brief intervals of dramatic change. There was a major transition about 50 years ago 
when pulmonary researchers such as Ben Burrows and Charles Fletcher ${ }^{1}$ recognized how pulmonary function and risk factors, especially cigarette smoke, were unifying features for the disparate clinical and radiographic manifestations of this syndrome. The contemporaneous discovery of alpha-1 antitrypsin deficiency (A1AT) provided a foothold for understanding COPD pathobiology and highlighted the potential importance of genetic predisposition. ${ }^{2}$ The concept of COPD which emerged at that time has evolved little over ensuing decades, though COPD prevalence and mortality continued to increase significantly. Looking back from 2014, we may now claim that another major transition is occurring, this one characterized by an explosion of understanding regarding the molecular and cellular abnormalities that underlie COPD. Pulmonary researchers deserve the credit for this progress, but it was also aided by a series of research initiatives supported by the National Heart, Lung, and Blood Institute (NHLBI), part of the National Institutes of Health (NIH), over the past decade. In parallel to these efforts, NHLBI has initiated and developed a successful program to promote COPD awareness across the United States. In this review, we will summarize those recent initiatives related to COPD and discuss how the NHLBI has used those initiatives to promote a balanced, interactive, and productive program of COPD research and education in the United States.

\section{Initiatives Launched by the NHLBI}

The vast majority of NHLBI funding for research is devoted to supporting grants initiated by individual investigators, but the Institute uses a small fraction of its funds for targeted programs. Institute-initiated programs, called initiatives can range from solicitation of Research Project Grant (Ro1) applications in a specific scientific area, to support for centers pursuing multiple, related projects, to the organization of coordinated programs that involve many grantee organizations, to the establishment of resources that provide specialized services to the research community. Initiatives may be supported by grant or contract mechanisms, and they can involve various levels of oversight and involvement by NHLBI program staff. As a general rule, NHLBI prefers to support research through investigator-initiated grants, and an initiative is considered only when there is a clear need, gap, or advantage to a more directed approach. For example, having a synchronized bolus of related studies can benefit investigators by allowing specialized peer review of their grant applications and by promoting cross-fertilization of ideas and collaborations among different laboratories working in a similar area. In other cases, it may be advantageous for NHLBI to provide leadership for the organization and operation of a program that involves multiple institutions, such as a clinical research network. Nearly all of the various models of initiatives have been used by NHLBI in recent years to foster COPD research. These initiatives have complemented investigator-initiated efforts and contributed to an increase in the amount of research on COPD and in the productivity of that research.

\section{Seeking Community Input}

NHLBI's strategic approach to COPD has been guided by ongoing conversations between the Institute and the community of COPD scientists and other key stakeholders. Important outputs of that dialogue were recommendations developed by two strategic Working Groups on COPD Research convened by NHLBI in $2001^{3}$ and 2002. 4 The first group discussed knowledge gaps and potential directions for future investigations, and the second specifically addressed needs and opportunities in clinical research. NHLBI followed these broadly themed Working Groups with a series of workshops that delved more deeply into topics raised by the initial 2 Working Groups. Those included therapeutic use of oxygen, COPD and lung cancer, genetics of COPD, case-finding strategies, COPD in never smokers, non-obstructive bronchitis, and primary prevention of chronic lung diseases. From these meetings, 4 general research needs emerged: (1) the need for better understanding of disease pathogenesis at the molecular and cellular levels, (2) the need for better classification of disease subtypes, with biomarkers for prognosis and prediction of therapeutic efficacy, (3) the need for easier access to human lung tissues from donors with COPD, and (4) the need for a clinical research network that can expeditiously and efficiently conduct clinical trials of promising but unproven treatments. Additionally, from discussions and meetings with various stakeholders, among them patients and patients' representatives, a fifth need emerged: the need for a public awareness and education campaign for COPD. These 5 needs and related specific recommendations have formed the basis for the implementation of an NHLBI strategy toward COPD research and education over the past decade. Particular initiatives contributing to that strategy are described below. 


\section{Lung Tissue Resources}

While animal models are useful for COPD research, full understanding of disease pathogenesis requires studies of lung tissues from COPD patients. ${ }^{3,4}$ The Working Groups from a decade ago emphasized this need and claimed that a significant body of knowledge from basic research was ready for translation into human studies. These groups also pointed out that COPD presents special challenges for human studies, since wide variations in the histological abnormalities and the heterogeneity of clinical presentations in this condition necessitate studies of relatively large numbers of well phenotyped participants. Ideally, these tissues would originate from a range of participants for which a rich set of clinical data have been collected, allowing investigators to confirm or refute the involvement in human disease of specific pathogenic processes suggested by animal models. A resource providing such human lung tissues and phenotypic data could also be used for discovery research, enabling analyses of human gene and protein expression, cellular phenotypes, and presence of microbial and/or viral infections at high spatial resolution.

In response to Working Group recommendations, NHLBI developed in 2004 the Lung Tissue Research Consortium (LTRC), ${ }^{5}$ whose purpose is to collect lung tissues, blood samples, X-ray computed tomographic (CT) scans, and other medical or exposure data, primarily from individuals with COPD or interstitial lung disease (ILD) as well as from control individuals. LTRC tissues derive from subjects' lung surgeries, which can be diagnostic biopsy, surgical lung resection for a suspected malignancy, lung explant, or lung volume reduction surgery (LVRS) for emphysema. In close to a decade of operation, the LTRC has collected tissues, blood, and data from nearly 3,000 individuals, and these can be requested through the LTRC website at no cost to the researcher.

Because the LTRC does not provide funding for research projects, NHLBI began a complementary program in 2008 to provide Small Grants for Lung Tissue Research. ${ }^{6}$ The goal of this funding opportunity announcement (FOA) was to enable small proof-of-concept projects using human tissues to initially test hypotheses developed using animal or in vitro model systems. Thirty-eight small grants (Ro3s) were awarded through this FOA, and many of those studies have now progressed to larger and more definitive studies of particular pathogenetic concepts. Overall, the LTRC has provided biospecimens and/or data in support of 173 research projects, enabling research in both COPD and ILD and playing a role in many of the translational studies ongoing in these fields.

\section{Initiatives to Promote Better Understanding of COPD Pathogenesis}

COPD is a complex disease with a multitude of phenotypes and disease outcomes including respiratory exacerbations, small airway disease, mucus hypersecretion, and the alveolar septal breakdown leading to emphysema. These phenotypes likely result from particular molecular and cellular events and pathways that can be better understood through studies of in vivo or in vitro model systems. The two strategic Working Groups described above highlighted a number of specific areas of research in COPD pathogenesis that deserved particular emphasis. ${ }^{3,4}$ In direct response to these and other recommendations, NHLBI developed initiatives to address mechanisms of mucous cell metaplasia and mucous secretion, ${ }^{7}$ causes and mechanisms of COPD exacerbations, ${ }^{8}$ novel biomarkers of COPD, ${ }^{9}$ and common pathogenetic mechanisms of lung cancer and $C O P D . .^{10}$ Research resulting from these initiatives, combined with investigator-initiated research, has led to numerous advances in the current basic science understanding of COPD pathogenesis. To recognize these significant changes, one needs only to look at how the appreciation of COPD pathogenesis from a decade ago ${ }^{11}$ has increased considerably in complexity, ${ }^{12}$ with the latter review including such topics as lipid mediators, aging, and growth factors and decreasing the prominence of proteases and antiproteases.

Working Groups on COPD have also emphasized the importance of using new understanding of pathogenic pathways for the development of novel therapeutics. To encourage such translational research, NHLBI launched a Specialized Centers of Clinically-Oriented Research (SCCOR) initiative that was specific for COPD. SCCORs were designed to foster interdisciplinary collaborations between the clinic and the bench and speed therapeutic applications of basic discoveries. Research supported by a COPD SCCOR ${ }^{13}$ using mouse models and human lung tissue led to the first indication of a molecular pathway linking respiratory viral infection to chronic lung disease, ${ }^{14,15}$ and animal research from another COPD SCCOR demonstrated that the angiotensin II receptor antagonist losartan, a drug commonly used to treat hypertension, may have the potential to influence emphysema progression. ${ }^{16}$ This observation has led to an NHLBI-funded Phase 2 clinical trial that is nearing completion (ClinicalTrials.gov Identifier: NCT00720226).

Finally, the NHLBI has encouraged the practical development of novel therapeutic agents based on molecular pathogenic understanding of lung disease through its 


\section{A Decade of NHLBI Programs Supporting COPD Research and Education}

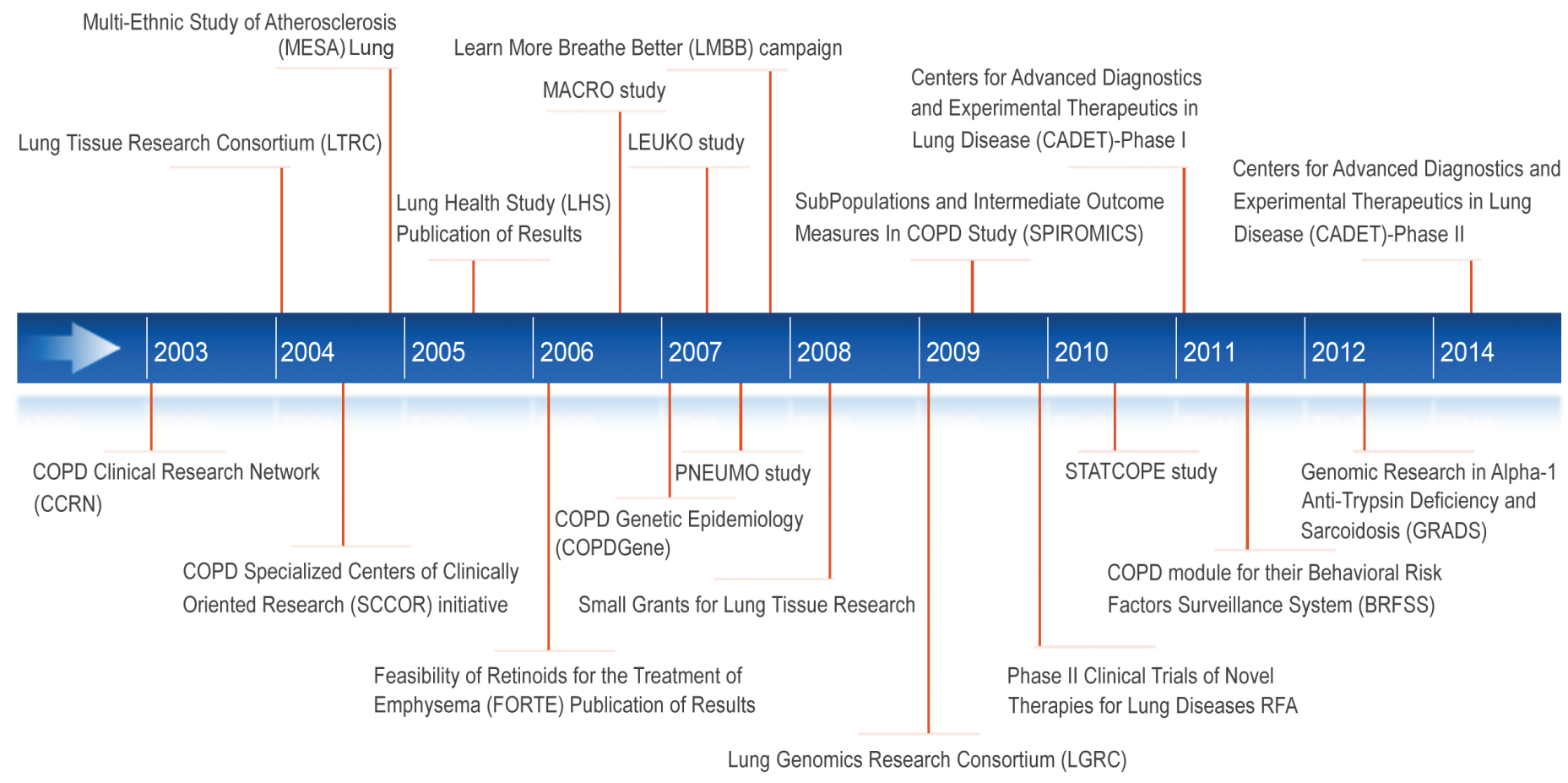

Figure 1

Centers for Advanced Diagnostics and Experimental Therapeutics in Lung Disease (CADET) program. CADET was designed in two stages: grants in the furst stage ${ }^{17}$ which funded research to identify and validate therapeutic targets, were awarded in 2011. A second group $^{18}$ of grants anticipated to be awarded in 2014 will support later, preclinical research on specific therapeutic products. Although research in this program is ongoing, CADET has already registered significant progress in a number of lung diseases including COPD. For example, one CADET grant showed that the chitinase-like glycoprotein YKL-4O may be a biomarker of lung function decline, particularly for smokers, ${ }^{19}$ while another identified a role for the mucin glycoprotein $\mathrm{MUC}_{5} \mathrm{~B}$ in mucociliary clearance in mouse models. ${ }^{20}$ Because smokers have significantly less MUC5B in their airways than healthy controls, ${ }^{21}$ it is possible that $\mathrm{MUC}_{5} \mathrm{~B}$ may be a new therapeutic target to promote airway defense in that population.

\section{COPD Genomics, Biomarkers, and Subpopulations}

The Working Groups recognized several related gaps in COPD research: a lack of data that could be used to classify COPD sub-types, insufficient understanding of genetic determinants of COPD, and the absence of biomarkers to track COPD progression. ${ }^{3,4}$ Progress in these areas typically requires cohort studies that collect clinical data and biological samples and then perform high throughput biospecimen analyses and high dimensional analyses of the data. The NHLBI responded with a number of programs to address these gaps. The Lung Genomics Research Consortium (LGRC) ${ }^{22}$ was funded in 2009 as part of the American Recovery and Reinvestment Act (ARRA). Its purpose was to collect genomic and gene expression data from LTRC samples. The resulting data combine the extensive clinical data collected on LTRC individuals with high throughput genomic analyses. The SubPopulations and Intermediate Outcome Measures In COPD Study (SPIROMICS), ${ }^{23}$ which was started in 2009 , is a multi-center observational study that will combine detailed clinical characterization with in-depth genomic analysis, imaging, and biomarker identification of over 3,000 individuals followed longitudinally for 3 years. ${ }^{24}$ The ultimate goal of SPIROMICS is to develop sub-classifications of COPD patients using molecular and imaging biomarkers. These sub-classifications may define groups amenable to specific, targeted clinical interventions. The study is ongoing, but SPIROMICS researchers have already begun to analyze biomarkers in plasma and serum. ${ }^{25}$ Finally, the Genomic Research in Alpha-1 Antitrypsin Deficiency and Sarcoidosis (GRADS) ${ }^{26}$ study, initially funded in 2012, is 
a multi-center study collecting clinical, genome and microbiome data on patients with 2 different types of rare chronic diseases which may affect the lung, A1AT, which predisposes patients to emphysema, and sarcoidosis.

While genetic components are known to contribute significantly to COPD risk, finding responsible genes had been confounded by small numbers of subjects and heterogeneous phenotypes. To identify genetic risk factors that had not yet been discovered, NHLBI funded a genome-wide association study (GWAS), called the COPD Genetic Epidemiology (COPDGene ${ }^{\circledR}{ }^{27}$ study in 2007. COPDGene is an investigator-initiated, multi-center study of over 10,000 non-Hispanic white and African American smokers with and without COPD. In addition to identifying genomic risk factors for COPD, COPDGene ${ }^{\circledR}$ is using state-of-the-art $\mathrm{CT}$ imaging to generate COPD sub-classifications. ${ }^{28}$ Over 65 articles resulting from the COPDGene ${ }^{\circledast}$ study have been published or are currently in press, and these address a range of topics, from new CT-based biomarkers for disease phenotype ${ }^{29}$ and acute exacerbation ${ }^{30}$ to genome-wide identification of risk loci for severe COPD. ${ }^{31}$ COPDGene ${ }^{\circledR}$ has sparked over 140 ancillary study proposals, at least 10 of which have been awarded NIH funding. Recently, NHLBI competitively renewed COPDGene ${ }^{\circledR}$ to study the progression of the disease at 5 years and to undertake whole-exome sequencing of the participants' genomes.

Lung researchers have also been taking advantage of NHLBI-funded cohort studies that are not primarily focused on lung diseases. For example, investigators have recruited participants from the Multi-Ethnic Study of Atherosclerosis (MESA) ${ }^{32}$ which was designed to study heart disease, to now investigate pulmonary disease in that well characterized cohort. By augmenting MESA data with pulmonary measures, investigators have shown that endothelial microparticles, suggestive of endothelial apoptosis, are elevated in individuals with COPD. ${ }^{33}$ These results hint at endothelial microparticles as a COPD biomarker that links endothelial cell apoptosis with COPD and emphysema. Other studies that are ancillary to existing clinical trials or cohort studies are supported through the NHLBI's Ancillary Studies in Clinical Trials requests for applications (RFA). ${ }^{34}$

\section{Clinical Trials}

The 2002 Working Group recommended a greater emphasis on clinical trials addressing potential COPD therapeutics. ${ }^{4}$ At that time, there were 3 major ongoing NHLBI-funded trials whose results were published after the Working Group met. The first was the National Emphysema Treatment Trial (NETT) (ClinicalTrials.gov
Identifier: NCTooooo606), which tested the effectiveness of LVRS on improving clinical outcomes. In 2003, the NETT trial published its conclusions that LVRS could improve exercise capacity in emphysema patients, but surgery decreased mortality when compared to conventional medical therapy only in a selected subset of patients. ${ }^{35}$ In 2005, the Lung Health Study (LHS) (ClinicalTrials.gov Identifier: NCTooo00568), whose primary results were released in $1994,{ }^{36}$ published the long-term effects of a smoking cessation intervention on 14.5 year mortality of smokers with minimal airway obstruction at the start of the study. The smoking cessation intervention, with only $22 \%$ maintained success rate, significantly decreased overall mortality, including mortality from respiratory disease. ${ }^{37}$ This was the first demonstration, by intent to treat analysis, that smoking cessation interventions can reduce mortality. Finally, the Feasibility of Retinoids for the Treatment of Emphysema (FORTE) (ClinicalTrials.gov Identifier: NCTooooo621) study pilot trial, published in 2006, found no observable clinical benefits to the administration of retinoids to patients with moderate-tosevere COPD. ${ }^{38}$

To respond to the growing need for randomized clinical trials for COPD patients, NHLBI established the COPD Clinical Research Network (CCRN) ${ }^{39}$ in $2003.4^{40}$ Its aim was to form a multi-center network that would perform multiple short-term clinical trials in individuals with COPD. CCRN trials conducted include: the PNEUMO study (ClinicalTrials.gov Identifier: NCTo0457977), a comparison of the responses of COPD patients to two different pneumococcal vaccines ${ }^{41}$; the LEUKO study (ClinicalTrials.gov Identifier: NCTo0493974), a test of the effect of the leukotriene modifier zileuton on COPD patient hospital length of stay for an exacerbation; ${ }^{42}$ and the MACRO study (Clinical Trials.gov Identifier: NCT00325897), an investigation into the effect of daily administration of the macrolide antibiotic azithromycin on exacerbation frequency. ${ }^{43}$ The latter study found a significant benefit of azithromycin use and is altering physician management of this disease. CCRN is currently completing the STATCOPE study (ClinicalTrials.gov Identifier: NCT01061671), whose purpose is to determine whether a statin has an effect on COPD exacerbation frequency or severity. Separate from the CCRN, NHLBI is also funding the Long-Term Oxygen Treatment Trial (LOTT) (ClinicalTrials.gov Identifier: NCTo0692198), ${ }^{44}$ to assess the efficacy of around-the-clock, supplemental oxygen therapy for patients with COPD and moderately severe hypoxemia. LOTT, which is expected to complete subject recruitment in late 2014, will provide a scientific basis for decisions regarding the clinical use of long-term oxygen 
treatment in COPD patients. Finally, NHLBI also funds several COPD clinical trials through its Phase II Clinical Trials of Novel Therapies for Lung Diseases RFA.45

In spite of these advances in clinical research, a large percentage of individuals affected by COPD, who could potentially benefit from new and established therapies, remain undiagnosed. In response to this problem, NHLBI convened a workshop in 2008 to address how best to improve COPD case-finding in the United States. ${ }^{46}$ Workshop participants agreed that a strategy was required and suggested that the combination of a questionnaire and measurement of peak expiratory flow might identify a high risk population in which medical evaluation, including spirometry, would be warranted. In 2011, NHLBI published an FOA entitled Development and Testing of a Case Finding Methodology in COPD, 47 and awardees are currently developing a case-finding tool in the hopes that it can be used both to improve the health of those unaware that they have COPD and to aid in the education and public awareness efforts described below.

\section{COPD Public Awareness and Education}

In 2006, NHLBI held discussions with a wide range of stakeholders to evaluate how to best address the needs of COPD research not only in the investigators' community, but also among patients and their health care providers. A major unmet need in COPD that came to light was the low level of awareness and understanding among the public at large about the then fourth (now third ${ }^{48}$ ) leading cause of death in this country, and the enormous public health burden this disease imposes on patients, their caregivers, and society at large. The NHLBI responded $^{49}$ in 2007 with the establishment of the Learn More Breathe Better (LMBB) campaign, ${ }^{50}$ which was the first national COPD awareness and education program. The LMBB has now been joined by more than 20 partner groups, including the COPD Foundation, the American Academy of Family Physicians, the American Lung Association, the American Thoracic Society, the American College of Chest Physicians, and the U.S. COPD Coalition. In 2009, the NHLBI convened a working group to assess and strengthen LMBB. Participants included various stakeholders including representatives from federal agencies, professional medical societies, and patient advocacy groups.

The goals of LMBB are to both raise broad public awareness of COPD and provide a sustained educational campaign to better inform and empower patients, families, and providers with the necessary information and tools to improve health outcomes. A couple of notable accomplishments of the LMBB campaign in- clude: 1) establishment of COPD education programs and development of state-wide COPD action plans in all 50 states, the District of Columbia, Puerto Rico, and Guam; and 2) development of a partnership with the Centers for Disease Control and Prevention (CDC) to develop a COPD module for their Behavioral Risk Factors Surveillance System (BRFSS). The COPD module in the BRFSS survey has been successfully implemented in 21 states, the District of Columbia, and Puerto Rico, and has provided valuable data on the prevalence of COPD at both state and community levels. The results of the first phase of the program were published in the Mortality and Morbidity Weekly Report. ${ }^{51}$ The LMBB program was recently evaluated and renewed by NHLBI to further enhance efforts to raise public awareness of COPD, continue to build alliances with strategic partners, and deliver greater focus on provider education and improved communication between patients and doctors. This program remains an essential component of the NHLBI strategy to translate research discoveries into improved health.

\section{Looking Ahead: The Next Decade NHLBI - Supported COPD Research and Education}

The significant scientific progress that has been made in COPD over the past decade promises to move this field into a new era characterized by the development and use of more effective therapeutics based on an understanding of molecular pathogenesis and selected for use in patients based on individual pathobiology. We are, hopefully, about to enter such a period of precision medicine for COPD. For this to occur, it is essential that the scientific community pursues further the research directions reflected in the NHLBI initiatives summarized above. Knowledge of the molecular mechanisms of disease pathogenesis garnered using animal models and human lung tissues from resources like the LTRC must be applied to stratify populations in studies such as SPIROMICS and COPDGene ${ }^{\circledR}$, translated into therapeutic products through efforts such as CADET, and tested in pragmatic and cost-effective clinical trials. It is also essential that the improved approaches be disseminated to patients and caregivers. Success with this strategy is within sight, but the distance yet to be travelled is huge. COPD is currently the third leading cause of death in the United States, with over 134,000 deaths annually. ${ }^{48}$ More than $6 \%$ of American adults have been told by a health care provider that they have this disease, and the geographic distribution of this condition shows ample 
variations, with highest prevalence in states clustered along the Ohio and lower Mississippi rivers. ${ }^{51}$ Much work remains to exploit the emerging advances in understanding COPD, to develop and test efficacious treatments, and to implement effective, individualized management approaches that will substantially improve the lives and outcomes of the millions of Americans afflicted by this disease. The vision of effective and individualized management will continue to drive our current research efforts, but the NHLBI is already looking beyond this anticipated period of molecular characterization and precision medicine and is preparing for yet another transition - one that will move pulmonary medicine into a period of prevention. We expect that future initiatives from NHLBI will begin to anticipate a transition toward that next geological period of COPD, one in which the impact of scientific research produces a substantial decrease in COPD prevalence and, perhaps, its eventual disappearance.

\section{Acknowledgements}

We thank Monique Ndenecho and Ann Taubenheim for their help in the preparation of this review.

\section{Declaration of Interest}

The authors have no financial interests to declare. 


\section{References}

1. Burrows B, Niden AH, Fletcher CM, Jones NL. Clinical types of chronic obstructive lung disease in London and in Chicago. A study of one hundred patients. Am Rev Respir Dis. 1964; 90:14-27.

2. Laurell CB, Eriksson S. The electrophoretic alpha-globulin pattern of serum in alpha-1-antitrypsin deficiency. Scand J Clin Lab Invest. 1963;15:132-140.

3. Croxton TL, Weinmann GG, Senior RM, Hoidal JR. Future research directions in chronic obstructive pulmonary disease. Am J Respir Crit Care Med. 2002;165(6):838-844.

4. Croxton TL, Weinmann GG, Senior RM, Wise RA, Crapo JD, Buist AS. Clinical research in chronic obstructive pulmonary disease: needs and opportunities. Am J Respir Crit Care Med. 2003;167(8):1142-1149.

5. National Heart, Lung and Blood Institute. Lung Tissue Research Consortium (LTRC). LTRC Web site. http://www.ltrcpublic.com/. Published 2008. Accessed March 6, 2014.

6. NHLBI. Small Grants for Lung Tissue Research, RFA-HL-08-008 Web site http://grants.nih.gov/grants/guide/rfa-files/RFA-HL-o8oo8.html. Published February 21, 2008. Accessed March 6, 2014.

7. NHLBI. Molecular Mechanisms of Mucous Cell Metaplasia and Excess Mucous Secretion in Human Airway Disease, RFA-HL-02-011 Web site. http://grants.nih.gov/grants/guide/rfa-files/RFA-HL-02011.html Published December 13, 2001. Accessed March 6, 2014.

8. NHLBI. Causes and Mechanisms of COPD Exacerbations, RFAHL-04-036 Web site. http://grants.nih.gov/grants/guide/rfa-files/ RFA-HL-04-036.html. Published September 16, 2004. Accessed March 6, 2014.

9. NHLBI. Novel Biomarkers of Chronic Obstructive Pulmonary Disease (COPD), RFA-HL-02-005 Web site.

http://grants.nih.gov/grants/guide/rfa-files/RFA-HL-02-005.html. Published July 21, 2001. Accessed March 6, 2014.

10. NHLBI. Common Pathogenetic Mechanisms of Lung Cancer and COPD, RFA-HL-11-002 Web site.

http://grants.nih.gov/grants/guide/rfa-files/RFA-HL-11-002.html. Published March 2, 2010. Updated August 6, 2010. Accessed March 6, 2014.

11. Barnes PJ, Shapiro SD, Pauwels RA. Chronic obstructive pulmonary disease: molecular and cellular mechanisms. Eur Respir J. 2003; $22(4): 672-688$.

12. Barnes PJ. Cellular and molecular mechanisms of chronic obstructive pulmonary disease. Clin Chest Med. 2014;35(1):71-86.

13. NHLBI. SCCOR in Chronic obstructive pulmonary disease (COPD), RFA-HL-05-008 Web site.

http://grants.nih.gov/grants/guide/rfa-files/RFA-HL-05-008.html. Published 2004. Accessed March 6, 2014.
14. Byers DE, Alexander-Brett J, Patel AC, et al. Long-term IL-33producing epithelial progenitor cells in chronic obstructive lung disease. J Clin Invest. 2013;123(9):3967-3982.

15. Kim EY, Battaile JT, Patel AC, et al. Persistent activation of an innate immune response translates respiratory viral infection into chronic lung disease. Nat Med. 2008 Jun;14(6):633-40.

16. Habashi JP, Judge DP, Holm TM, et al. Losartan, an AT1 antagonist, prevents aortic aneurysm in a mouse model of Marfan syndrome. Science. 2006;312(5770):117-121.

17. NHLBI. Centers for Advanced Diagnostics and Experimental Therapeutics in Lung Diseases Stage I (CADET I), RFA-HL-11-015 Web site. http://grants.nih.gov/grants/guide/rfa-files/RFA-HL-11015.html. Published Feb 26, 2010. Accessed March 6, 2014.

18. NHLBI. RFA-HL-14-001; Centers for Advanced Diagnostics and Experimental Therapeutics in Lung Diseases Stage II (CADET II), RFA-HL-14-001 Web site. http://grants.nih.gov/grants/guide/rfa-files/ RFA-HL-14-001.html. Published 2013. Accessed March 6, 2014 March 6.

19. Guerra S, Halonen M, Sherrill DL, et al. The relation of circulating YKL-40 to levels and decline of lung function in adult life. Respir Med. 2013;107(12):1923-1930.

20. Roy MG, Livraghi-Butrico A, Fletcher AA, et al. Muc5b is required for airway defence. Nature. 2014;505(7483):412-416.

21. Innes AL, Woodruff PG, Ferrando RE,et al. Epithelial mucin stores are increased in the large airways of smokers with airflow obstruction. Chest. 2006;130(4):1102-1108.

22. Lung Genomics Research Consortium (LGRC). LGRC Web site. http://www.lung-genomics.org/lgrc. Published 2010. Accessed March 6, 2014.

23. NHLBI, University of North Carolina at Chapel Hill. SubPopulations and InteRmediate Outcome Measures In COPD Study (SPIROMICS) Web site. http://www.cscc.unc.edu/spir/ Accessed March 6, 2014.

24. Couper D, Lavange LM, Han M, et al. Design of the Subpopulations and Intermediate Outcomes in COPD Study (SPIROMICS). [Published online ahead of print 2013 Sep 12]. Thorax. doi:10.1136/thoraxjnl-2013-203897.

25. O'Neal WK, Anderson W, Basta PV, et al. Comparison of serum, EDTA plasma and P100 plasma for luminex-based biomarker multiplex assays in patients with chronic obstructive pulmonary disease in the SPIROMICS study. J Transl Med. 2014;12(1):9. doi:10.1186/1479-5876-12-9.

26. NHLBI. Genomics Research in Alpha-1 Antitrypsin Deficiency and Sarcoidosis (GRADS) study Web site. https://www.gradslung.org/ index.html . Published 2014. Accessed March 6, 2014.

27. COPD Genetic Epidemiology.COPDGene study Web site. http://www.copdgene.org/.

Accessed March 6, 2014 March. 
28. Regan EA, Hokanson JE, Murphy JR, et al. Genetic epidemiology of COPD (COPDGene) study design. COPD. 2010;7(1):32-43.

29. Galban CJ, Han MK, Boes JL, et al. Computed tomography-based biomarker provides unique signature for diagnosis of COPD phenotypes and disease progression. Nat Med. 2012;18(11):1711-1715.

30. Wells JM, Washko GR, Han MK, et al. Pulmonary arterial enlargement and acute exacerbations of COPD. N Engl J Med. 2012 ;367(10): 913-921.

31. Cho MH, McDonald ML, Zhou X, et al. Risk loci for chronic obstructive pulmonary disease: a genome-wide association study and meta-analysis. Lancet Respir Med. 2014; 2(3): 214-225.

32. NHLBI, Multi-Ethnic Study of Atherosclerosis (MESA)Coordinating Center. MESA Web site. http://www.mesa-nhlbi.org/. Published 2014. Accessed March 6, 2014.

33. Thomashow MA, Shimbo D, Parikh MA, et al. Endothelial microparticles in mild chronic obstructive pulmonary disease and emphysema. The Multi-Ethnic Study of Atherosclerosis Chronic Obstructive Pulmonary Disease study. Am J Respir Crit Care Med. 2013.188(1):60-68. doi: 10.1164/rccm.201209-1697OC.

34. NHLBI. Ancillary Studies in Clinical Trials, RFA-HL-14-004 Web site. http://grants.nih.gov/grants/guide/rfa-files/RFA-HL. Published September 28, 2012. Updated September 25, 2013. Accessed March 6, 2014 .

35.Fishman A, Martinez F, Naunheim K, et al. A randomized trial comparing lung-volume-reduction surgery with medical therapy for severe emphysema. N Engl J Med. 2003;348(21):2059-2073.

36.Anthonisen NR, Connett JE, Kiley JP, et al. Effects of smoking intervention and the use of an inhaled anticholinergic bronchodilator on the rate of decline of FEV1. The Lung Health Study. JAMA.1994; 272(19):1497-1505.

37. Anthonisen NR, Skeans MA, Wise RA, Manfreda J, Kanner RE, Connett JE. The effects of a smoking cessation intervention on 14.5-year mortality: a randomized clinical trial. Ann Intern Med. 2005;142(4):233-239.

38. Roth MD, Connett JE, D'Armiento JM, et al. Feasibility of retinoids for the treatment of emphysema study. Chest. 2006;130(5): $1334-1345$.

39. COPD Clinical Research Network (CCRN) Web site. http://www.copdcrn.org/ Accessed March 6, 2014.

40. Albert RK. The NHLBI COPD clinical research network. Pulm Pharmacol Ther. 2004;17(3):111-112.
41. Dransfield MT, Nahm MH, Han MK, et al. Superior immune response to protein-conjugate versus free pneumococcal polysaccharide vaccine in chronic obstructive pulmonary disease. Am J Respir Crit Care Med. 2009 ;180(6):499-505.

42. Woodruff PG, Albert RK, Bailey WC, et al. Randomized trial of zileuton for treatment of COPD exacerbations requiring hospitalization. COPD. 2011;8(1):21-29.

43. Albert RK, Connett J, Bailey WC, et al. Azithromycin for prevention of exacerbations of COPD. N Engl J Med. 2011;365(8):689-698.

44. NHLBI. Long-term Oxygen Treatment Trial (LOTT) study Web site. http://jhuccs1.us/lott/. Published 2009. Updated Feburary 24, 2014. Accessed March 62014.

45. NHLBI.Phase II Clinical Trials of Novel Therapies for Lung Diseases, RFA-HL-12-022 Web site.

http://grants1.nih.gov/grants/guide/rfa-files/RFA-HL-12022.html. Published March 7, 2011. Accessed March 6, 2014.

46. NHLBI . NHLBI Workshop--A Case-finding Strategy for Moderateto-Severe COPD in the United States.http://www.nhlbi.nih.gov/ meetings/workshops/case-finding-exesum.htm. Published November 2009. Accessed March 10, 2014.

47. NHLBI. RFA-HL-12-011; Development and Testing of a Case Finding Methodology in COPD, RFA-HL-12-011 Web site. http://grants1.nih.gov/ grants/guide/rfa-files/RFA-HL-12-011.html. Published March 17, 2011. Accessed March 102014.

48. Centers for Disease and Prevention (CDC). Underlying cause of death 1999-2010 on CDC WONDER online database. CDC WONDER Web site. http://wonder.cdc.gov/ucd-icd1o.html. Published 2011. Accessed February 7, 2014.

49. NHLBI. NIH Launches New Campaign Aimed at Increasing Awareness, Early Diagnosis and Treatment of $\operatorname{COPD}$ [news release]. http://www.nhlbi.nih.gov/news/press-releases/2007/ nih-launches-new-campaign-aimed-at-increasing-awareness-earlydiagnosis-and-treatment-of-copd.html. Published January 18, 2007. Accessed March 7, 2014.

50. NHLBI. Learn More Breathe Better (LMBB) Web site. http:// www.nhlbi.nih.gov/health/public/lung/copd/lmbb-campaign/. Accessed March 6, 2014.

51. CDC. Chronic obstructive pulmonary disease among adults United States, 2011. MMWR. 2012;61(46):938-943. 